\title{
VIRTBot: exploring chatbot design for promoting scientific initiatives
}

\author{
Thomas Lombardi, University of the Virgin Islands, thomas.lombardi@uvi.edu \\ Matt North, Utah Valley University,mnorth@uvu.edu \\ N. Brice Orange, OrangeWave Innovative Science,LLC, orangewaveno@gmail.com \\ Kisha Coulanges, University of the Virgin Islands, kisha.coulanges@students.uvi.edu \\ Jace Johnson, Utah Valley University, jacejerryjohnson@gmail.com
}

\begin{abstract}
Chatbots have proven to be effective tools in the fields of marketing, sales, customer relationship management and many other applications. This research explores the opportunities for chatbots to contribute to the promotion of scientific research and initiatives. The Etelman Observatory Research Center of the University of the Virgin Islands (UVI) houses the Virgin Islands Robotic Telescope (VIRT), a fully-automated, robotically controlled, and queue-driven 0.5 meter research grade telescope. The Etelman Observatory's mission is to be a world-class research and education center that engages with the local community through various outreach activities in all its initiatives. Given the challenges of physical presence during the COVID-19 crisis, Observatory personnel decided to adopt chatbot technology to engage interested parties over the Internet in its key scientific instrument for astrophysics -- VIRT. VIRTBot is a chatbot designed to provide VIRT with a voice that interested community members can engage with directly. The team implemented VIRTBot with Amazon Web Services (AWS) technologies in the cloud and deployed the solution online. Volunteers were surveyed about their knowledge of the Observatory's activities after reviewing either an FAQ or engaging with VIRTBot. The study demonstrated that the FAQ outperformed VIRTBot in terms of knowledge dissemination, but VIRTBot outperformed the FAQ in measures of interest and engagement. Our research suggests that, under the right conditions, chatbots improve engagement over traditional web resources in promoting STEM educational initiatives to the public.
\end{abstract}

Keywords: Chatbot, Amazon Web Services

\section{Introduction}

At a time when scientific research has become more vital to public policy than ever before, the importance of that research has become more difficult to explain to the public. While this phenomenon most obviously underscores current events related to the COVID-19 pandemic, more modest scientific ventures struggle with communicating their relevance and contributions to a distant public as well. The Etelman Observatory Research Center of the University of the Virgin Islands (UVI) is one such institution. This research facility supports a diverse collection of scientific, technology development, education, and outreach initiatives while serving as an astronomical research station (Orange et al., 2021). In 2020, Etelman Observatory personnel from OrangeWave Innovative Science, LLC (OWIS), who oversee operations, approached our team to assist with communicating the facility's mission to the public. The purpose of this research is to provide data and analysis for guiding Etelman Observatory's promotional activities to the public. 


\section{Issues in Information Systems}

Volume 22, Issue 4, pp. 69-82, 2021

Located in the U.S. Virgin Islands, the Etelman Observatory (Figure 1) is the home of the Virgin Islands Robotic Telescope (VIRT), a 0.5 meter research-grade telescope that fills an observational void between mainland U.S. and European observatories (Morris, 2020). The relatively small design of VIRT (Figure 2) was specific to its main scientific goal to provide rapid optical follow-up of transient astronomical events (transients) known as Gamma-ray bursts (Giblin et al., 2003). Gamma-ray bursts (or GRBs) are some of the most powerful explosions in the Universe. In addition to this astronomical research program, the Observatory is a key research center and engineering training component in UVI's Physics and Engineering programs and supports STEM education, climate monitoring throughout the USVI, small-scale agriculture, biological research and tourism (Orange et al., 2021). Despite its many contributions to the intellectual and economic interests of the USVI, its commitment to be a vibrant venue for the community, tourism, and STEM enrichment activities has been stymied by the COVID-19 pandemic. As such, Observatory personnel engaged our team to help promote its activities to the public at a time when COVID-19 regulations in the territory precluded more conventional outreach activities like public observing nights and tours.

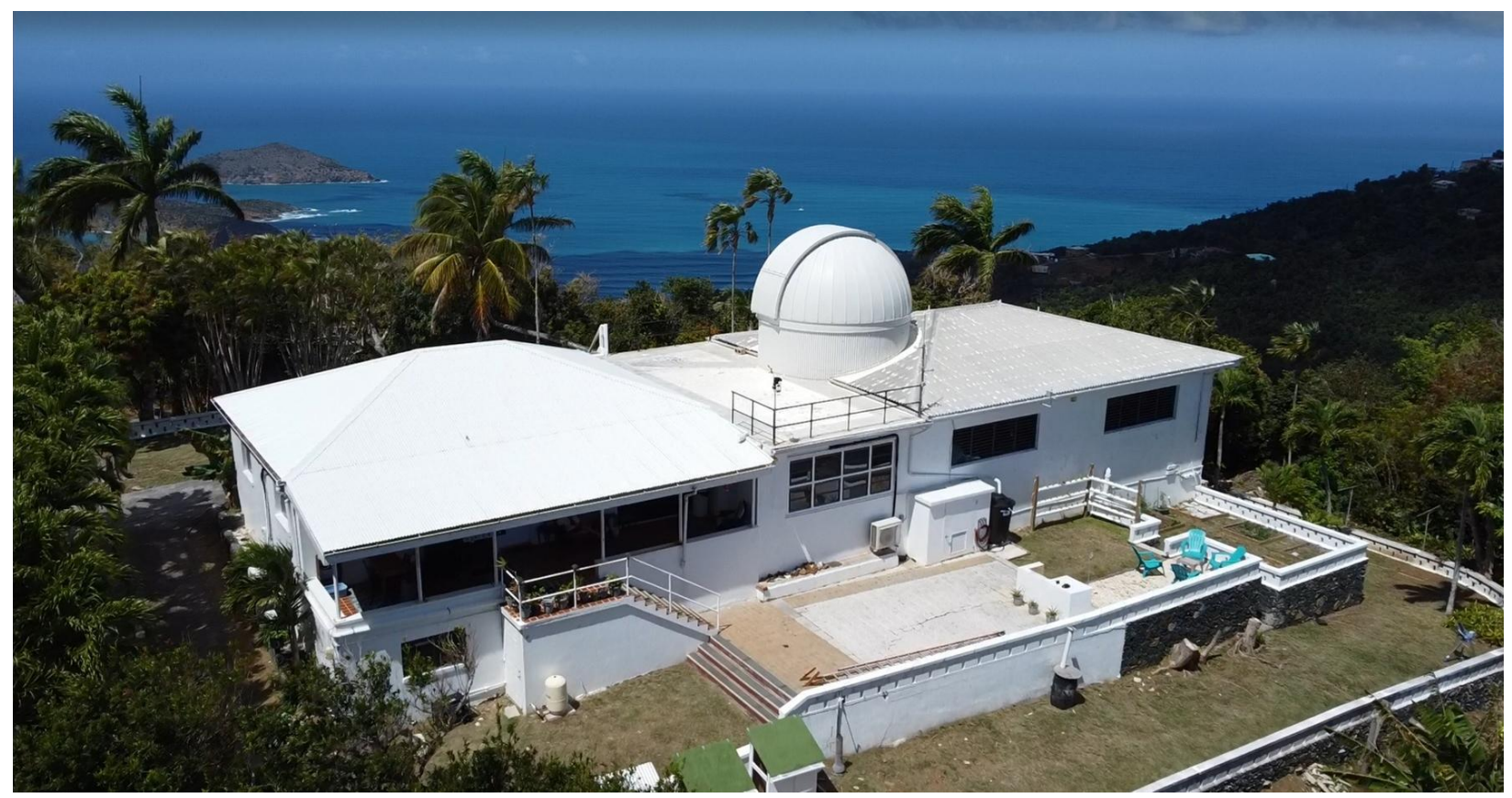

Figure 1: Etelman Observatory, St. Thomas, U.S. Virgin Islands

Many businesses have adopted chatbots for applications in a wide variety of industries (Chaves \& Gerosa, 2020). Chatbot technology capitalizes on recent trends in artificial intelligence, machine learning, cloud computing and big data analytics to create a realistic agent that can interact autonomously with end users to share information and accomplish tasks. Recent forecasts predict that the use of chatbots is likely to increase in fields such as healthcare, online retail, and hospitality ("Chatbot Market - Growth, Trends, COVID-19 Impact, and Forecasts (2021-2026)", n.d.). In addition to this growing list of business applications, chatbots have also demonstrated their value in educational contexts such as advising and tutoring (Gonda, Luo, Wong \& Lei, 2018; Moraes Neto \& Fernandes, 2019; Ondas, Pleva \& Hladek, 2019). Moreover, chatbot solutions are particularly well-suited to the uncertainties arising from the COVID-19 pandemic. From this perspective, the joint team of University of the Virgin Islands and Utah Valley University researchers proposed VIRTBot, a chatbot designed to speak for VIRT, as a potential technical and business solution to the promotional and STEM engagement challenges of Etelman Observatory. 


\section{Issues in Information Systems}

Volume 22, Issue 4, pp. 69-82, 2021

Cloud computing infrastructures such as Amazon Web Services (AWS) facilitate the design and deployment of chatbots in a relatively short period of time. Since OWIS personnel were already familiar with the AWS platform due to their use of AWS EC2 Instances (Virtual Machines in the AWS environment), AWS proved to be the most efficient and effective means of addressing their promotional and educational needs. The base functionality for a chatbot is provided by an AWS CloudFormation template in JSON which provisions the underlying resources for the conversational agent including Amazon Lex, AWS Lambda and S3 storage resources ("Q\&A Chatbot Self-Paced Guide", 2021). The CloudFormation stack includes an administrative interface for configuring the domain-level knowledge in astronomy as well as the general-purpose knowledge such as manners to improve the user experience. VIRTBot captures the relevant information for outreach and educational purposes to provide a "voice" for VIRT who speaks for Etelman Observatory.

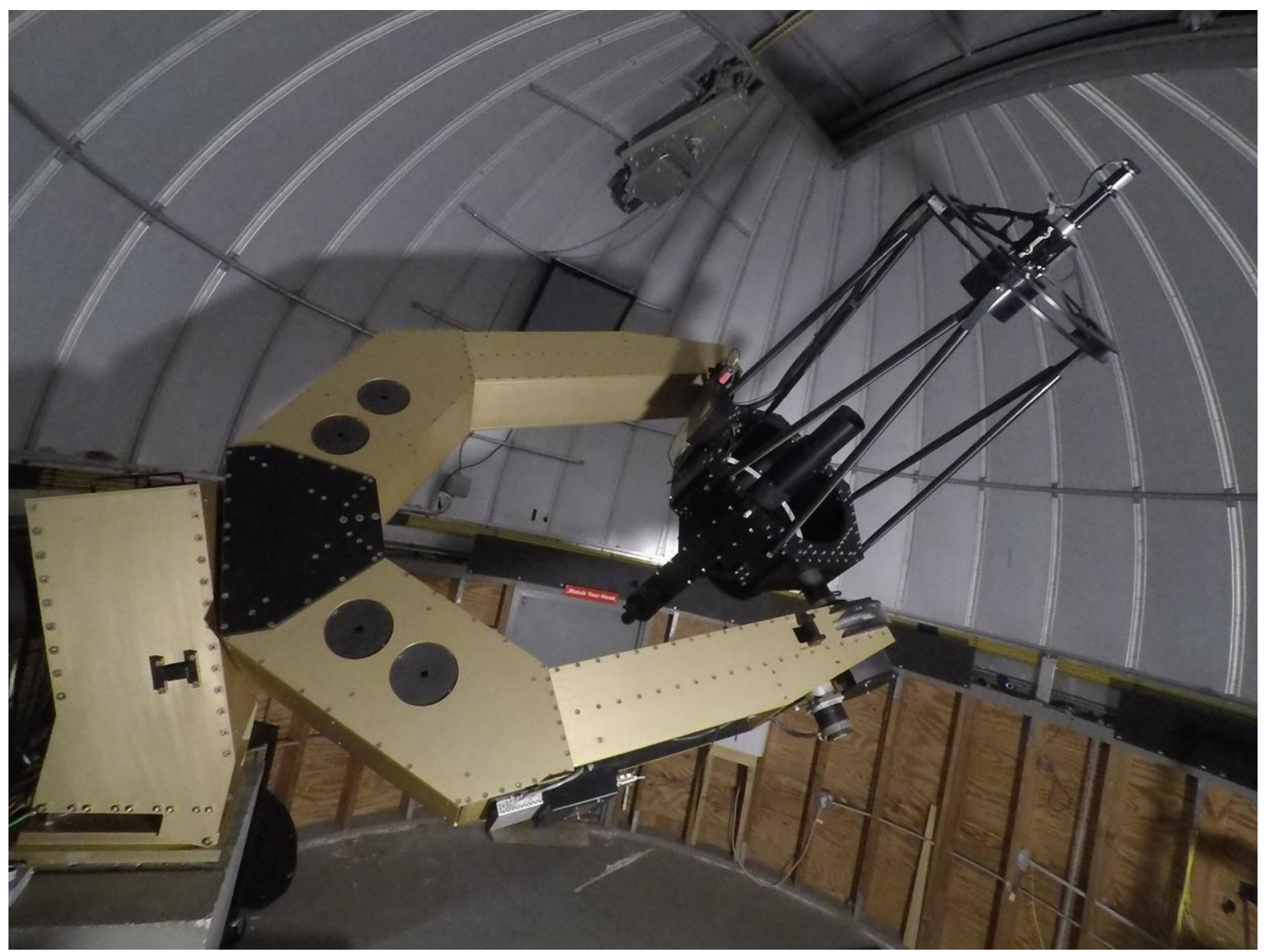

Figure 2: The Virgin Islands Robotic Telescope

VIRTBot offers the Etelman Observatory an opportunity to test the effectiveness of chatbots as outreach tools for the sciences. The primary hypothesis is that the interaction provided by VIRTBot will increase the retention of vital outreach and educational information. Secondarily, we expect that the chatbot will increase the level of interest experienced by users over more direct methods of information exchange such as an FAQ web page. The experiment recruited Utah Valley University student volunteers and randomly assigned each volunteer to one of two groups. The first group reviewed an FAQ website with the relevant promotional and educational information. The second group interacted with VIRTBot which was configured with the same promotional and educational information. After completing their review of the 


\section{Issues in Information Systems}

Volume 22, Issue 4, pp. 69-82, 2021

material provided, each volunteer completed a survey quizzing the participant's recognition of the relevant content. The team analyzed the results to verify if there was a statistically significant difference in the comprehension of the relevant information between the two groups. In this way, the research seeks to answer the question: Is VIRTBot an effective outreach tool or just a fun distraction?

\section{Literature Review}

The use of computerized chatbots to automate interactions with humans could reasonably be traced back to early imitation game Turing tests in the 1950s, and chatbot technologies as they currently exist have been developing for at least the past two decades (Adamopoulou \& Moussiades, 2020). The current state-of-theart includes the melding of artificial intelligence, machine learning and expert systems to create an artificial responder that approximates human accuracy, interactivity, and even emotion as closely as possible (Sameera \& Woods, 2015). Limitations of time, money and expertise have all served to incentivize organizations - for-profit and non-profit alike- to investigate the possibilities offered through the use of chatbots (Shumanov \& Johnson, 2021). Chatbots can be available 24 hours a day, every day of the year, can remember everything they are taught and even learn new things, and generally cost less than their human counterparts (Yorita, et al., 2019).

\section{Why chatbots?}

In recent years, chatbots have grown in popularity in education (Smutny, 2020). Chatbots offer the potential to increase engagement and enhance interactivity in education, information dissemination, and other areas (Moraes Neto \& Fernandes, 2019). Chatbots have been effective in distance education because they have the ability to reach an online audience. Furthermore, chatbots have the ability to generate interest and engagement by enhancing course teaching (Gonda et al., 2018).

Chatbots are gaining popularity not only in education, but also in business. This is because in an online platform chatbots can inform users about product features (Ondas, Pleva \& Hladek, 2019). Possible reasons as to why chatbots are growing in popularity within corporations and education is their immediate availability which means that companies can utilize a chatbot to fill various assistant, secretary, and information desk roles for companies during and after company hours (Niharika, 2020).

Chatbots can ultimately save companies money by reducing the amount of customer support representatives that are needed on the clock after normal workday hours. Additionally, chatbots can reduce the amount of customer support representatives within a company because they can take on the bulk of customer questions (Lindsay, 2017). Chatbots also reduce the amount of human error within companies because the AI can be designed to always answer specific questions with perfect memory (Hussain, Sianaki \& Ababneh, 2019).

\section{The need for "humanness"}

In order to increase the effectiveness of chatbots, and thereby justify their use, the development of anthropomorphic characteristics is a key consideration (Westerman, Cross \& Lindmark, 2019). Chatbots that merely lend voice to a list of questions and answers have been found to be less effective, while those possessing more dynamic capabilities produce more committed interactions with end users and are generally viewed as more useful and effective (Pricilla, Lestari \& Dharma, 2018).

Shumanov and Johnson (2021) found that frequently asked questions (FAQ) documents are often largely ignored as a source of information for web site users, even when those users are looking for information that is contained within available FAQ resources. For chatbots to overcome this, they need to be more personalized and interactive with their users. This finding is supported by findings in Sameera and Woods 


\section{Issues in Information Systems}

Volume 22, Issue 4, pp. 69-82, 2021

(2015), as well as Chaves and Gerosa (2020). Research has consistently found that when chatbots are engaging and responsive, the human beings interacting with them perceive them to be more accurate (Sameera \& Woods, 2015) as well as more helpful (Chaves \& Gerosa, 2020). Thus, projects seeking to improve the user experience by supplanting or supplementing FAQs with chatbot technology would do well to ensure that these attributes are present. This is easier said than done.

Despite significant improvements in chatbot technology over the past five years, infusing chatbots with appropriate, timely and effective emotive responses remains a challenge (Moraes Neto \& Fernandes, 2019). Emulating human responses requires perception of affective intent coupled with appropriate reaction to context cues (Araujo, 2018). Automating computerized versions of such reactions has occupied much scholarly attention for decades, with continuous improvement still needed. Some such improvements have already proven to increase chatbot acceptance (Ramesh et al., 2017).

\section{Chatbots in the context of our research}

Our research in this paper includes both improving upon information transfer via chatbots rather than traditional FAQs and enriching the user experience in the process. This objective is common for chatbot use (Hussain, Sianaki \& Ababneh, 2019). We seek to use chatbots to promote information dissemination about the VIRT telescope at the Etelman Observatory, and to encourage interested parties to engage more immersively with the telescope and the observatory's educational and scientific offerings.

Enhancement of educational objectives and outcomes has long been a subject of chatbot research (Adamopoulou \& Moussiades, 2020). Research by Ondas, Pleva and Hladek (2019) found that learners were more engaged and achieved higher assessment outcomes when the instruction they received was augmented through the use of thoughtful, well-designed chatbot technology. Findings from Moraes Neto and Fernandes (2019) were similar, with specific positive outcomes when learners interacted with chatbots in distance learning environments. Since most people who will interact with the chatbot in our research here will not have the opportunity to travel to the Virgin Islands to see the Etelman Observatory in person, Moraes Neto and Fernandes' research is of particular interest to us. Based partially on their work, in the present study we hypothesized that the chatbot we have developed for this research will yield significantly better experiences and outcomes for our users than a static FAQ page.

Because part of the mission of the Etelman Observatory is to educate and to provide astronomical research resources, we seek to emulate the outcomes of research that explored ways in which chatbots have demonstrably improved learning. Gonda, et al. (2018) proposed applying seven "good teaching" principles to chatbot design. We have sought to include these principles in our development of VIRTBot, with the specific objective of maximizing the educational impact the chatbot has on those who interact with it. We further sought to keep the chatbot's design as simple and intuitive as possible, in harmony with the recommendations made by Baez et al. (2020). By following these established and recommended principles in our own work, we seek to realize similar results to those achieved by previous researchers (Yorita et al., 2019).

\section{Methodology}

Our study focused on the following research questions:

RQ1) Does the use of a chatbot increase the retention of knowledge related to VIRT and Etelman Observatory over less expensive methods of communication such as a web page? 


\section{Issues in Information Systems}

Volume 22, Issue 4, pp. 69-82, 2021

RQ2) Does the use of a chatbot increase the level of interest in VIRT and Etelman Observatory over less expensive methods of communication such as a web page?

To operationalize the research questions, the research team outlined the core scientific and promotional facts from the Etelman Observatory perspective including the Etelman Observatory's location, VIRT's purpose, VIRT's characteristics, and common activities at the Etelman Observatory. After reviewing the copy with subject matter experts on the team, the core scientific and promotional facts were captured in both a web page FAQ and VIRTBot. By analyzing the differences in user responses to the same information in these two different media, the study aimed to capture the value added, if any, by VIRTBot.

The research hypotheses to be tested are as follows:

$\mathrm{H}_{1}$ : VIRTBot significantly increases the retention of knowledge related to VIRT and the Etelman Observatory over an FAQ web page.

$\mathrm{H}_{2}$ : VIRTBot significantly increases the level of interest in VIRT and the Etelman Observatory over an FAQ web page.

To test these hypotheses, the researchers divided volunteers into two groups with the task of learning about Etelman Observatory online. The first group (FAQ Group) was asked to learn about Etelman Observatory by reading the FAQ page on Etelman Observatory's website: https://eo.uvi.edu/faq.html. The second group (BOT Group) endeavored to learn about Etelman Observatory by interacting with VIRTBot through a web interface: https://rshkllkx3g.execute-api.us-east-1.amazonaws.com/prod/static/client.html. After reviewing their respective resources, each participant completed a survey comprising nine multiple choice/multiple answer questions and one open-ended question appropriate to their group. 


\section{Issues in Information Systems}

Volume 22, Issue 4, pp. 69-82, 2021

Table 1: Survey Questions

\begin{tabular}{|c|c|c|}
\hline Question \# & FAQ Group Questions & BOT Group Questions \\
\hline 1 & Where is Etelman Observatory/VIRT? & Where is Etelman Observatory/VIRT? \\
\hline 2 & $\begin{array}{l}\text { What is the purpose of VIRT? (Select all that } \\
\text { apply) }\end{array}$ & $\begin{array}{l}\text { What is the purpose of VIRT? (Select all that } \\
\text { apply) }\end{array}$ \\
\hline 3 & What kind of telescope is VIRT? & What kind of telescope is VIRT? \\
\hline 4 & $\begin{array}{l}\text { Besides scientific research and STEM } \\
\text { education, what other activities does Etelman } \\
\text { Observatory support? (Select all that apply) }\end{array}$ & $\begin{array}{l}\text { Besides scientific research and STEM education, } \\
\text { what other activities does Etelman Observatory } \\
\text { support? (Select all that apply) }\end{array}$ \\
\hline 5 & How easy was it to use the FAQ? & How easy was it to use VIRTbot? \\
\hline 6 & $\begin{array}{l}\text { How quickly were you able to find answers in } \\
\text { the FAQ for the four questions about the } \\
\text { Etelman Observatory and VIRT? }\end{array}$ & $\begin{array}{l}\text { How accurate were the responses that VIRTbot } \\
\text { gave you? }\end{array}$ \\
\hline 7 & $\begin{array}{l}\text { How likely would you be to share this FAQ } \\
\text { with children who are learning about } \\
\text { telescopes or astronomy? }\end{array}$ & $\begin{array}{l}\text { How likely would you be to share VIRTbot with } \\
\text { children who are learning about telescopes or } \\
\text { astronomy? }\end{array}$ \\
\hline 8 & $\begin{array}{l}\text { How likely would you be to share the Etelman } \\
\text { Observatory's FAQ with an educator? }\end{array}$ & $\begin{array}{l}\text { How likely would you be to share VIRTBot with } \\
\text { an educator? }\end{array}$ \\
\hline 9 & $\begin{array}{l}\text { How likely would you be to use the Etelman } \\
\text { Observatory's FAQ or web site for your own } \\
\text { interests? }\end{array}$ & $\begin{array}{l}\text { How likely would you be to use VIRTBot for your } \\
\text { own interests? }\end{array}$ \\
\hline 10 & $\begin{array}{l}\text { Do you have any other feedback, such as } \\
\text { recommendations to improve the Etelman } \\
\text { Observatory's FAQ? }\end{array}$ & $\begin{array}{l}\text { Do you have any other feedback, such as } \\
\text { recommendations to improve VIRTbot, } \\
\text { observations and ideas, or feature } \\
\text { recommendations? }\end{array}$ \\
\hline
\end{tabular}

Table 1 summarizes the questions posed to participants in the study. Questions 1 through 4 measure the participants' knowledge of important details related to the Etelman Observatory. We test hypothesis 1 by checking for significant differences between the responses of the two groups on questions 1 through 4 with a Chi-square test of independence. Questions 7 - 9 measure the participants' interest in the Etelman Observatory as presented in the given modality (web page FAQ or chatbot). Significant differences between the responses of the two groups for questions 7 - 9 (one-tailed T-test) could suggest that VIRTBot increases the level of interest in the Etelman Observatory and its work. Questions 5, 6 and 10 gather user experience data which is not directly related to the hypotheses in the study, but serve as useful feedback should the Etelman Observatory choose to continue the VIRTBot project beyond this pilot study.

\section{Results}

The survey results include data from 55 respondents (24 in the FAQ group; 31 in the BOT group) out of 147 total invitations (response rate $37.4 \%$ ) sent to students at Utah Valley University. 


\section{Issues in Information Systems}

Volume 22, Issue 4, pp. 69-82, 2021

\section{Hypothesis 1}

$\mathrm{H}_{1}$ : VIRTBot significantly increases the retention of knowledge related to VIRT and the Etelman Observatory over an FAQ web page.

The collected data did not support hypothesis 1. The Chi-square test of independence for Questions 1, 3, and 4 showed that there was no significant association between the assigned group and the responses to these questions. For the most extreme example, every respondent answered Question 3 correctly regardless of their group assignment. The Chi-square test of independence showed no significant association between group assignment and responses to Question $1, X^{2}(1, N=55)=0.13206, p=0.7163$, and Question 4, $X^{2}(1$, $\mathrm{N}=55)=1.3768, \mathrm{p}=0.2407$. In short, our results did not falsify the null hypothesis for these questions and therefore we find no evidence of a statistically significant association between group assignment and knowledge retention in these cases.

Where we do identify a significant relationship between group assignment and knowledge retention, the relationship is in the opposite direction of the stated hypothesis: The Chi-square test of independence for Question 2 showed that there was a significant association between the assigned group and the responses to this question, $\mathrm{X}^{2}(1, \mathrm{~N}=55)=4.616, \mathrm{p}=0.03167$. The evidence supports the notion that the FAQ group demonstrated increased retention of the knowledge over VIRTBot in this case. Only 3 respondents in the BOT group could correctly answer Question 2 compared to 9 respondents in the FAQ group.

A Welch Two-Sample T-Test found that the two groups also had significant differences in the perceived accuracy of the FAQ and VIRTBot. The 24 participants in the FAQ group $(M=1.833, S D=1.308)$ compared to the 31 participants in the BOT group $(M=2.613, S D=1.054)$ demonstrated significantly lower accuracy ratings $(1=$ Extremely accurate $)$ as expressed in Question $6, t(43.526)=-2.3819, p=$ 0.01083 . Figure 3 summarizes these differences visually. Not only did the respondents in the BOT group have more trouble answering one of the knowledge-specific questions than their counterparts in the FAQ group, but also many of the BOT group respondents recognized failures of accuracy in the VIRTBot tool.

These findings are consistent with some of the unstructured feedback provided by the respondents. Some of the respondents in the BOT group highlighted these accuracy problems in the survey's open-ended question (Question 10). In fact, four respondents mentioned Question 2 specifically as posing problems. A particularly succinct example explained: "When I typed in 'What is the purpose of VIRT?' It did not understand the question". Based on these results, the use of a chatbot did not increase the retention of knowledge related to VIRT and Etelman Observatory over less expensive methods of communication such as a web page. The Discussion section of the paper proposes some tentative explanations for the observed results. 
FAQ Group Accuracy Ratings

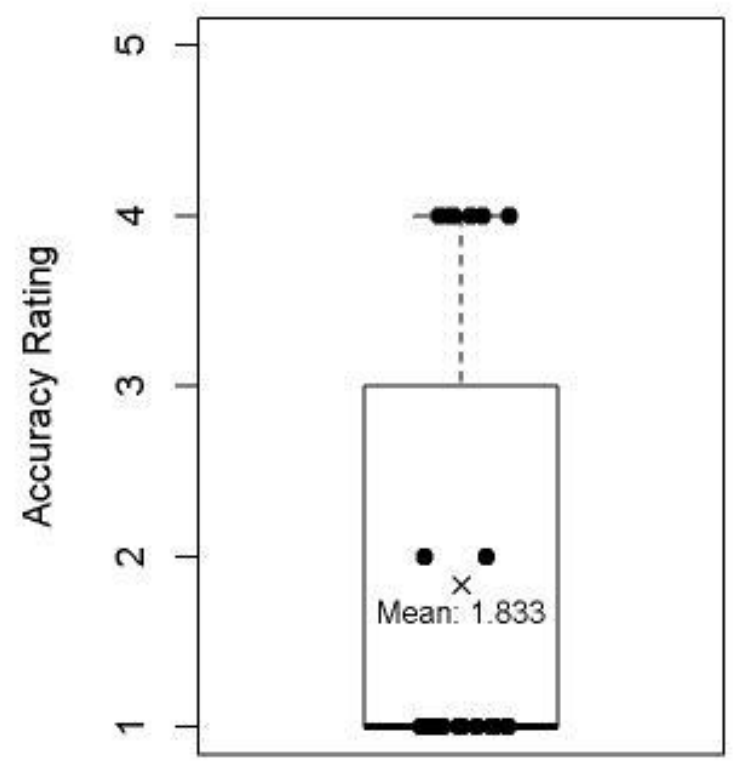

\section{BOT Group Accuracy Ratings}

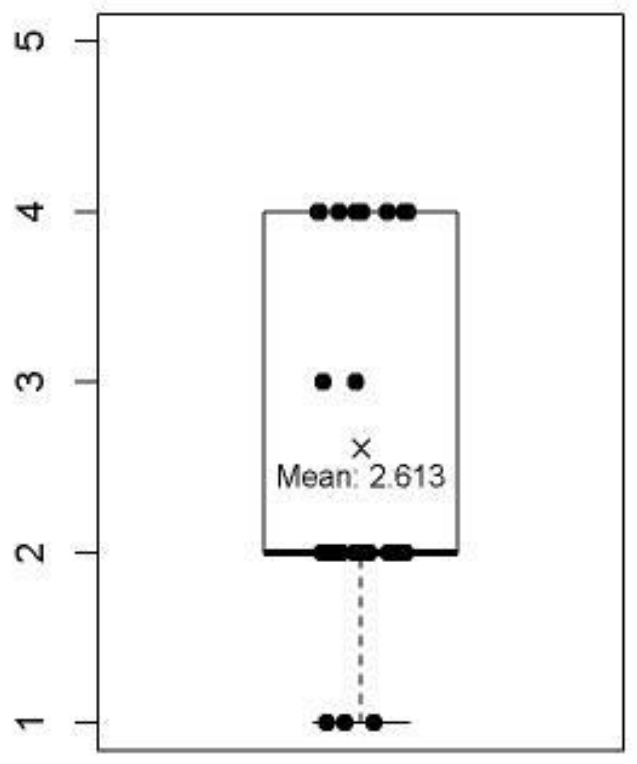

Figure 3: Accuracy Ratings (1 = Extremely accurate; 5 = Extremely inaccurate)

\section{Hypothesis 2}

$\mathrm{H}_{2}$ : VIRTBot significantly increases the level of interest in VIRT and the Etelman Observatory over an FAQ web page.

The collected data did support hypothesis 2 as demonstrated with T-Tests on Questions 7 and 8. A Welch Two-Sample T-Test found that the two groups had significant differences in the likelihood of referring children to their assigned resource. The 24 participants in the FAQ group $(M=3.083, S D=1.442)$ compared to the 31 participants in the BOT group $(M=2.226, S D=1.087)$ demonstrated significantly higher ratings $(1=$ Extremely likely $)$ as expressed in Question $7, t(41.512)=2.428, p=0.0098$. In other words, VIRTBot users were significantly more likely to refer the chatbot to children learning this subject matter. Figure 4 summarizes the results related to Question 7. 


\section{FAQ Group Child Ratings}

\section{BOT Group Child Ratings}
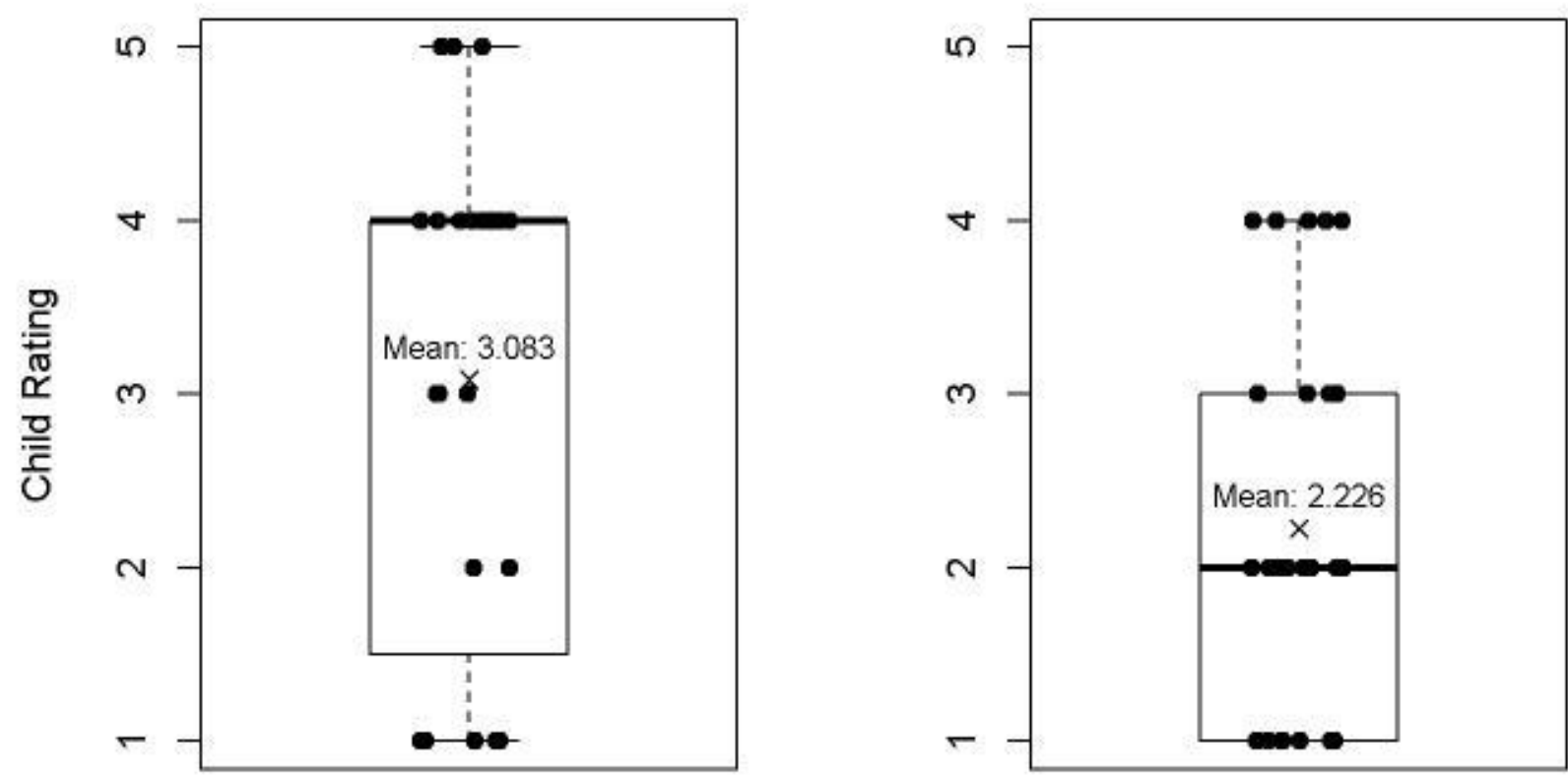

Figure 4: How likely would you be to share VIRTBot with Children (1=Extremely likely; 5=Extremely unlikely)

Similarly, a Welch Two-Sample T-Test found that the two groups had significant differences in the likelihood of referring educators to their assigned source of information. The 24 participants in the FAQ group $(M=2.583, S D=1.381)$ compared to the 31 participants in the BOT group $(M=1.935, S D=0.727)$ demonstrated significantly higher ratings $(1=$ Extremely likely) as expressed in Question $8, t(32.787)=$ $2.0858, p=0.02243$. In other words, VIRTBot users were significantly more likely to refer the chatbot to educators teaching this subject matter. Figure 5 summarizes the results related to Question 8.

To round out the results section, we note that neither Question 5 (ease of use) nor Question 9 (personal interest) demonstrated significant differences between the groups. Regarding Question 5, there was no significant effect for ease of use, $t(40.413)=-0.041156, p=0.9674$, despite the fact that the FAQ group $(M$ $=2.083, S D=1.348)$ reported slightly lower scores ( 1 = Extremely easy) than the BOT group $(\mathrm{M}=2.0973$, $\mathrm{SD}=0.978$ ). This result is particularly encouraging as it suggests that VIRTBot users did not find the system significantly more difficult to use than a web page for knowledge dissemination. On the other hand, the results for Question 9 demonstrate that VIRTBot users were not more likely to use VIRTBot for their own interests than were the FAQ group to use the FAQ web page. There was no significant difference for the likelihood to pursue the topic, $t(41.514)=-0.08834, p=0.93$, between the FAQ group $(M=3.29, S D=$ $1.429)$ and the BOT group $(M=3.323, S D=1.077)$.

Overall, the results provide some support to the notion that the use of a chatbot increases the level of interest in VIRT and Etelman Observatory over less expensive methods of communication such as a web page. Broadly speaking, VIRTBot users were significantly more likely to refer the tool to children and educators than FAQ users. At the same time, VIRTBot users did not report different levels of concern about its ease of use as compared to the FAQ group. And yet, we recognize that VIRTBot did not significantly increase a user's likelihood to pursue the topic further with the tool. At least one user expressed excitement about VIRTBot and offered a feature improvement: "It is an excellent tool. I would recommend that when someone types, it will suggest the question." The Discussion section will pursue these themes in additional detail. 
FAQ Group Teacher Ratings

\section{BOT Group Teacher Ratings}
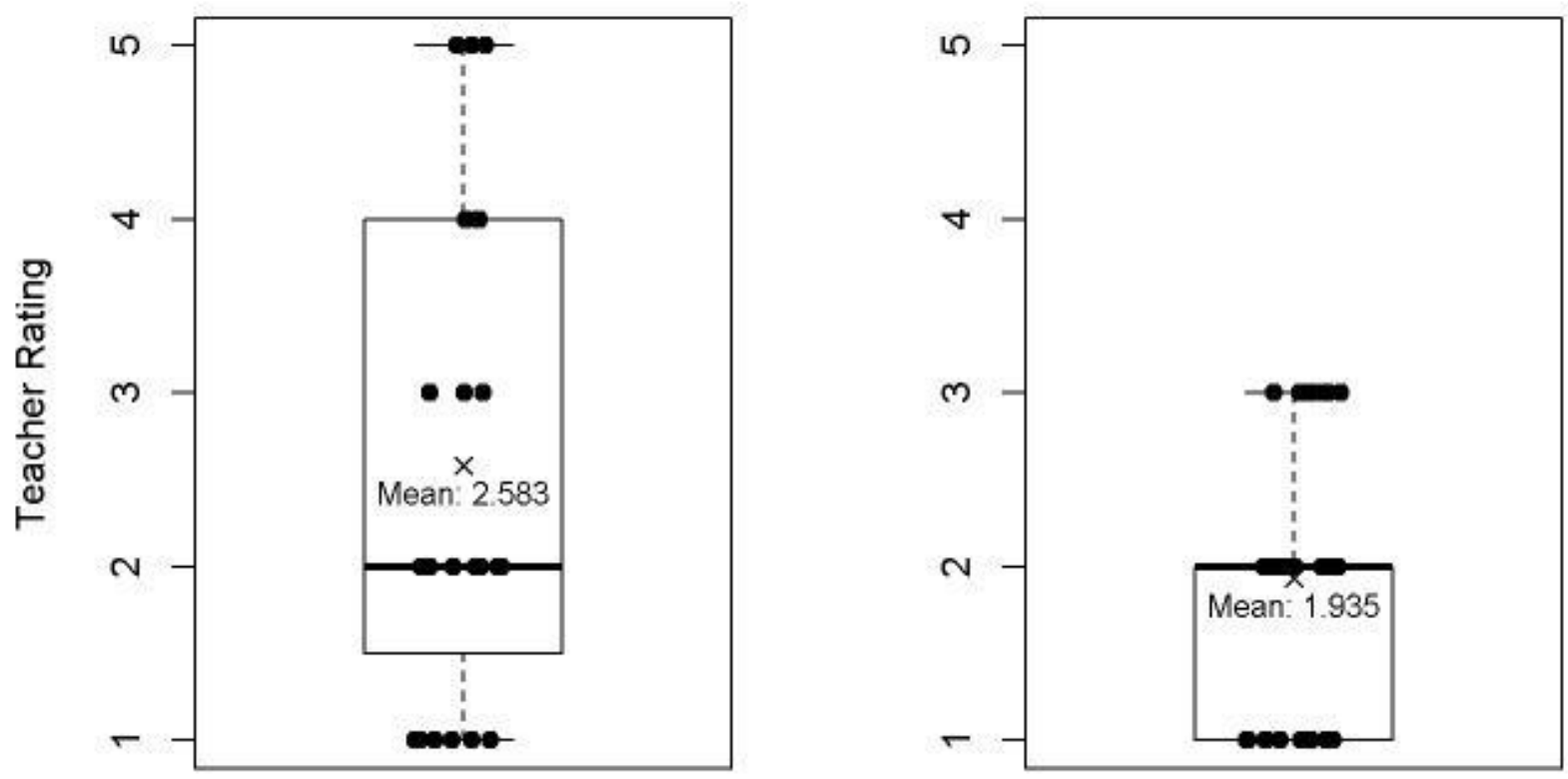

Figure 5: How likely would you be to share VIRTBot with Educators (1=Extremely likely; 5=Extremely unlikely)

\section{Discussion}

\section{Limitations of the study}

Our survey pool consisted of a convenience sample of just under 150 people. Without enticements, our response rate was reasonably good, and the convenience sample did include people from diverse backgrounds, geographic regions and other personal attributes. We intentionally did not gather specific data regarding demographics or locations. We are confident that the group of respondents to our survey was meaningfully diverse, however we acknowledge that the number of responses $(\mathrm{N}=55)$ and the lack of specific personal attribute information does limit our findings. In fact, power analysis confirms that our sample size is small given the significance level (0.05) and effect size with the power ranging between 0.65 for Question 2 and 0.77 for Question 7). Our study may also not be generalizable to a large number of organizations that may be interested in trying chatbots as a means of educational engagement. Our subject matter is specifically one observatory with very specific equipment, uses, and objectives and our study was designed to address those.

\section{Future directions}

This study raises numerous questions which could be pursued as continued lines of inquiry. The results of our hypothesis tests showed that our chatbot was not as effective and accurate as we thought it would be, resulting in significantly lower scores when compared to FAQs. In an age when an increasing portion of technology is shifting toward dynamic, interactive interfaces, one would expect that a chatbot would be more desirable and useful when compared to a traditional FAQ. Yet our chatbot did not realize this outcome. Was it the design of the chatbot, insufficient artificial intelligence capabilities, an ineffective user interface, 


\section{Issues in Information Systems}

Volume 22, Issue 4, pp. 69-82, 2021

or some other limitation(s) that resulted in a failure to confirm our initial hypotheses? Further research must be conducted to determine the answer to this question.

Beyond just the hypotheses tested in this study, our broader objective is to find engaging ways to promote education via internet-enabled and remote interaction with resources such as the Etelman Observatory and its robotic telescope. Will adding chatbots to the observatory's website draw in and connect with a broader audience of students and educators, or is time better spent implementing other technologies? Would it be more engaging and interesting to create web-based or app-based viewers to experience the telescope's observations from afar? What would be the optimal range of technologies that would attract and retain a robust audience of users? Regarding the results of this current study, some survey respondents indicated that they simply copied and pasted some of the survey questions into VIRTBot to see what response they might get. Why did they resort to this method of interaction with the chatbot? Was it because their own attempts using natural language failed to generate a useful response, or was it a default reaction to being asked specific questions? Much research could yet be done solely on questions of why people interact with chatbots the way they do.

With respect to the business objectives of Etelman Observatory, the research presented in this study was the first step in developing a cost/benefit analysis. Is the benefit of implementing a chatbot worth the cost as compared to other options? Amazon Web Services relies on a pay as you go model that incurs cost to the business based on application usage. Therefore, it is critical for a business to understand the benefits of chatbot technology, if any, prior to their deployment. Based on this pilot study, the cost of VIRTBot is about $\$ 50$ per month with some variation based on usage. Future work will continue to refine the required inputs for such analysis.

\section{Conclusion}

Returning to the question from our introduction, it might be tempting to dismiss VIRTBot as a fun distraction given our results. It is always interesting when research fails to confirm a hypothesis that seems like it would be true. At the outset of our research, all of the authors on this paper agreed that we believed that a chatbot would be significantly and demonstrably more effective and interesting than a traditional, static FAQ. While some qualitative comments from our survey respondents indicated interest in, and support for the chatbot technology, the actual responses to most of our questions actually showed that the FAQ was more often preferred and effective for disseminating information about the Etelman Observatory and its robotic telescope. In order to reach a point where our findings may change to confirm our hypotheses, it is clear that chatbot technology must continue to improve to increase both accuracy and user experience.

\section{Acknowledgements}

T.L., N.B.O, and K.C. acknowledge financial support from NASA EPSCoR award 80NSSC19M0060. N.B.O. also acknowledges financial support from NASA MUREP MIRO award NNX15AP95A, the University of the Virgin Islands' Greatness Through Innovation award 260380, and South Carolina Space Grant award NNX15AL49H. K.C. also acknowledges financial support from OrangeWave Innovative Science, LLC.

\section{References}

Adamopoulou E. \& Moussiades, L. (2020). Chatbots: History, technology, and applications. Machine Learning with Applications, 2, 100006. 


\section{Issues in Information Systems}

Volume 22, Issue 4, pp. 69-82, 2021

Araujo, T. (2018). Living Up to the Chatbot Hype: The influence of anthropomorphic design cues and communicative agency framing on conversational agent and company perceptions. Computers in Human Behavior, 85, 183-189.

Baez, M., Daniel, F., Casati, F. \& Benatallah, B. (2020). Chatbot Integration in Few Patterns, in IEEE Internet Computing, doi: 10.1109/MIC.2020.3024605.

Chatbot Market - Growth, Trends, COVID-19 Impact, and Forecasts (2021-2026). (n.d.). Retrieved April 18, 2021, from https://www.mordorintelligence.com/industry-reports/chatbotmarket.

Chaves, A.P. \& Gerosa, M.A. (2020). How Should My Chatbot Interact? A Survey on Social Characteristics in Human-Chatbot Interaction Design. International Journal of HumanComputer Interaction, DOI: 10.1080/10447318.2020.1841438.

Giblin, T.W., Neff, J.E., Hakkila, J., Drost, D.M., \& Andreasian-Thomas, N. (2003). Development of a Gamma-Ray Burst Automated Response and Remote $0.5 \mathrm{~m}$ Telescope at Etelman Observatory at the University of the Virgin Islands. HEAD meeting \#7 American Astronomical Society Meeting, 35,624 .

Gonda, D. E., Luo, J., Wong, Y. \& Lei, C. (2018). Evaluation of Developing Educational Chatbots Based on the Seven Principles for Good Teaching. 2018 IEEE International Conference on Teaching, Assessment, and Learning for Engineering (TALE), Wollongong, NSW, 446-453, doi: 10.1109/TALE.2018.8615175.

Hussain S., Sianaki O. A. \& Ababneh N. (2019). A Survey on Conversational Agents/Chatbots Classification and Design Techniques. In: Barolli L., Takizawa M., Xhafa F., Enokido T. (eds) Web, Artificial Intelligence and Network Applications. WAINA 2019. Advances in Intelligent Systems and Computing, vol 927.

Lindsay, D. P. (2017). Chatbot Chatter. American Society for Quality Control, 50(7), 3-6.

Moraes Neto, A. J., \& Fernandes, M. A. (2019). Chatbot and Conversational Analysis to Promote Collaborative Learning in Distance Education. 2019 IEEE 19th International Conference on Advanced Learning Technologies (ICALT), 324-326.

Morris, D.C. (2020). The University of the Virgin Islands B.S. with a Concentration in Astronomy: Developing Careers in STEM through Astrophysics. 2020 235th American Astronomical Society Meeting, 52, 117.03.

Niharika, M., Keerthana, K. R., Yeshwanth Prasad, B. U. (2020). The Role of Chatbots in Enhancing Staff Productivity of Network Service Providers in Bengaluru. IUP Journal of Organizational Behavior, 19(4) 7-21.

Ondas, S., Pleva, M., \& Hladek, D. (2019). How chatbots can be involved in the education process. 2019 17th International Conference on Emerging E-Learning Technologies and Applications (ICETA), $575-580$. 


\section{Issues in Information Systems}

Volume 22, Issue 4, pp. 69-82, 2021

Orange, N.B., Gokuldass, P., Lombardi, T., \& Parsons, M. (2021). The Etelman Observatory and the Virgin Islands Robotic Telescope: A Pivotal and Resilient Educational Center and Research Instrument. 2021 237th American Astronomical Society Meeting, 53, 139.02.

Pricilla, C., Lestari, D.P. \& Dharma, D. (2018). Designing Interaction for Chatbot-Based Conversational Commerce with User-Centered Design. 5th International Conference on Advanced Informatics: Concept Theory and Applications (ICAICTA), Krabi, 244-249.

Q\&A Chatbot Self-Paced Guide. (2021). Retrieved 18 April 2021, from https://d14r4b0p45o2hl.cloudfront.net/guides/qnachatbot/index.html

Ramesh K., Ravishankaran S., Joshi A., Chandrasekaran K. (2017) A Survey of Design Techniques for Conversational Agents. In: Kaushik S., Gupta D., Kharb L., Chahal D. (eds) Information, Communication and Computing Technology. ICICCT 2017. Communications in Computer and Information Science, vol 750. Springer, Singapore. https://doi.org/10.1007/978-981-10-65446_31

Sameera A. A. \& Woods, J. (2015). Survey on Chatbot Design Techniques in Speech Conversation Systems. International Journal of Advanced Computer Science and Applications, 6(7), 215-220.

Shumanov, M. \& Johnson, L. (2021). Making Conversations with Chatbots more Personalized. Science Direct, 117, https://doi.org/10.1016/j.chb.2020.106627.

Smutny, P., Schreiberova, P. (2020). Chatbots for learning: A review of educational chatbots for the Facebook Messenger. Elsevier Ltd, vol 151. https://doi.org/10.1016/j.compedu.2020.103862

Westerman, D., Cross, A. C., \& Lindmark, P. G. (2019). I Believe in a Thing Called Bot: Perceptions of the Humanness of "Chatbots." Communication Studies, 70(3), 295-312.

Yorita, A., Egerton, S., Oakman, J., Chan, C. \& Kubota, N. (2019). Self-Adapting Chatbot Personalities for Better Peer Support. International Conference on Systems, Man and Cybernetics. 10.1109/SMC.2019.8914583. 